\title{
Overhanging interfacial gravity waves of large amplitude
}

\author{
By D. I. MEIRON AND P. G. SAFFMAN \\ Applied Mathematics, California Institute of Technology, Pasadena, CA 91125
}

(Received 30 August 1982)

Methods to investigate the existence of overhanging gravity waves of permanent form at the interface between two uniform fluids of different density are discussed. Numerical results which demonstrate their existence are presented.

\section{Introduction}

We consider irrotational solutions of the Euler equations of motion which describe nonlinear travelling waves of permanent form at the interface between immiscible inviscid fluids of different densities $\rho_{1}$ and $\rho_{2}$ in the presence of gravity when $\rho_{1}>\rho_{2}$ and $\rho_{1}$ is the density of the lower fluid. For $\rho_{2}=0$ and in the absence of interfacial tension these solutions describe the well-known surface waves of permanent form (Stokes waves). It was conjectured by Stokes that these waves achieve their maximum amplitude when the maximum velocity of the fluid in the $x$-direction equals the phase speed of the wave. It follows that in a frame moving with the phase speed the highest surface wave would have stagnation points at the crests, and from a local analysis of the equations of motion Stokes showed that the crests would be sharp and enclose an angle of $120^{\circ}$. The existence of these sharp waves has subsequently been shown rigorously (Amick, Toland \& Fraenkel 1982). Note that since $\rho_{2}=0$ there is no physical inconsistency associated with the infinite velocity as the stagnation point is approached from above.

For $\rho_{2}>0$ the interfacial waves of permanent form between fluids otherwise at rest were studied extensively by Holyer (1979), who applied the method of Padé approximants to sum computer-extended perturbation series in the wave height. In this case, a limiting solution could no longer possess a stagnation point, in a frame moving with the wave, on the interface, as this would imply an infinite velocity in one of the fluid layers.

Despite the inconsistency associated with a stagnation point it is, however, still conceptually possible for the fluid speed in the $x$-direction relative to fluid far away to equal the phase speed at some point on the interface. The surface then becomes vertical at this point. Holyer proposed that the wave amplitude at which such a vertical tangent arises corresponds to the highest-possible interfacial wave. Such a criterion is clearly attractive from two standpoints. Firstly, it includes the criterion used in the case of Stokes waves. Secondly, the profile of any interfacial wave whose $x$-velocity exceeded its phase speed at some point would have an S-shape in which heavy fluid lies above light fluid. This situation is of course statically unstable, and should be dynamically unstable to at least disturbances of sufficiently short wavelength. However, instability and existence are separate questions, and it was pointed out by Saffman \& Yuen (1982) that there is no dynamical or kinematical reason to reject such overturned waves as solutions to the equations for steady waves. In this paper we present evidence for the existence of overturned steady interfacial 
waves. Such waves were explicitly calculated using two distinct numerical methods. The first is the potential plane approach of Saffman \& Yuen, in which the physical coordinates of the interface are computed as functions of the potential and stream function. The second is the dipole-sheet approach of Baker, Meiron \& Orszag (1982), which yields a nonlinear integral equation for the interface configuration. We describe both methods briefly in $\S 2$ and present the numerical results in $\S 3$.

\section{Formulation}

We are considering gravity waves at the interface between two incompressible, inviscid, irrotational fluids of different densities. With no loss of generality, we set the gravitational acceleration $g=1$ and the wavelength to $2 \pi$. The physical variables associated with the upper fluid are denoted by a subscript 2 and those of the lower fluid by a subscript 1 .

In a frame moving with the wave the problem of determining the shape of the interface may be posed as finding the solution to the equations:

$$
\begin{gathered}
\psi_{1}(x, y(x))=0, \quad \psi_{2}(x, y(x))=0, \\
B+\rho_{1}\left(y(x)+\frac{1}{2}\left(\nabla \phi_{1}\right)^{2}\right)=\rho_{2}\left(y(x)+\frac{1}{2}\left(\nabla \phi_{2}\right)^{2}\right), \\
\phi_{1} \sim-c x \quad \text { as } \quad y \rightarrow-\infty, \\
\phi_{2} \sim-c x \quad \text { as } \quad y \rightarrow \infty .
\end{gathered}
$$

Here $\psi_{1,2}$ and $\phi_{1,2}$ are harmonic functions which denote the stream functions and velocity potentials of the fluid layers, $B$ is the Bernoulli constant and $c$ is the phase speed. Rather than solve for $\phi$ and $\psi$ as functions of the physical coordinates, it is preferable to solve numerically for the coordinates $x, y$ as functions of the variables $\phi, \psi$. Note that since $x, y$ are parametrized in terms of the potentials, this method is appropriate for the calculation of overturned waves. A finite system of equations is obtained by truncating the modal expansion of $x$ and $y$ and satisfying the equations at a finite number of points. Further details may be found in Saffman \& Yuen (1982).

A second approach follows from the fact that the interfacial wave may be recast as the solution to a nonlinear integral equation. In a stationary frame of reference, Baker et al. (1982) have shown that the wave profile and fluid velocities satisfy the equations

$$
\begin{gathered}
u \dot{x}=v \dot{y} \\
u \frac{\dot{\mu}}{\dot{x}}=-2 A\left[\frac{1}{2} u^{2}+\frac{1}{2} v^{2}+\frac{1}{8}\left(\frac{\dot{\mu}^{2}}{\dot{x}^{2}+\dot{y}^{2}}\right)+y\right]+K
\end{gathered}
$$

where denotes differentiation with respect to a parameter $\alpha(0 \leqslant \alpha \leqslant 2 \pi), u, v$ are the average $x$ - and $y$-components of velocity across the interface in wave-fixed coordinates, $c$ is the phase speed, $K$ is a constant to be determined, and $A$ is the Atwood ratio defined by

$$
A=\frac{\rho_{1}-\rho_{2}}{\rho_{1}+\rho_{2}}
$$

The dipole-sheet strength $\mu(\alpha)$ is related to the velocities and the shape of the interface through the Biot-Savart law:

$$
\begin{aligned}
u(\alpha)-i v(\alpha)=-c+\frac{1}{4 \pi i} \int_{0}^{2 \pi} \frac{\left[\dot{\mu}\left(\alpha^{\prime}\right)(\dot{x}(\alpha)+i \dot{y}(\alpha))-\dot{\mu}(\alpha)\left(\dot{x}\left(\alpha^{\prime}\right)+i \dot{y}\left(\alpha^{\prime}\right)\right)\right]}{\dot{x}(\alpha)+i \dot{y}(\alpha)} & \quad \times \cot \frac{1}{2}\left[\left(x(\alpha)+i y(\alpha)-\left(x\left(\alpha^{\prime}\right)+i y\left(\alpha^{\prime}\right)\right)\right] d \alpha^{\prime}\right.
\end{aligned}
$$


The unknown variables in $(2.5-2.8)$ are $x(\alpha), y(\alpha), \mu(\alpha), c$ and $K$. The specification of the parameter $\alpha$ is at one's disposal. For the calculation of interfacial waves where $y$ is a single-valued function of $x$ one can set

$$
x(\alpha)=\alpha .
$$

However, in the calculation of waves with a vertical tangent, $x(\alpha)$ is not monotonic, and it is necessary to employ a representation in which

$$
\dot{x}\left(\alpha^{*}\right)=0
$$

at the points of vertical tangency $\alpha^{*}$. We shall restrict attention to symmetrical waves with the erest at $x=0$ and the trough at $x=\pi$. There are then in general two such points in $0<\alpha<\pi$ for an overhanging wave. Since for an overhanging wave $\dot{x}$ becomes negative in a region about $\alpha=\alpha^{*}$ and $u\left(\alpha^{*}\right)=0$, a natural choice for the parametric representation is

$$
\begin{aligned}
& \dot{x}(\alpha)=\frac{u(\alpha)}{\frac{1}{\pi} \int_{0}^{\pi} u\left(\alpha^{\prime}\right) d \alpha^{\prime}}, \\
& x(0)=0 .
\end{aligned}
$$

For small waves where $|u| \approx c,(2.11)$ reduces to (2.9). (It is conceivably possible for the denominator in (2.11) to vanish since the integral does decrease as the wave amplitude increases. However, for the results reported here the denominator is nonzero, and (2.11) is a valid form.) Then equations (2.5)-(2.8), (2.11)-(2.12) form a nonlinear system of equations for the shape of the wave. These are discretized using a spectral method and collocation at equally spaced values of $\alpha$, and the resulting equations solved by Newton's method. Further details of implementation may be found in Baker et al. (1982).

\section{Results}

The numerical accuracy of the two methods was checked for each waveheight by comparison of runs at various resolutions, and also for the potential method by monitoring the decay of the coefficients in the modal expansion. The agreement between the two schemes was very close for waves that were not too steep and for which each method appeared accurate. It was observed that the potential-plane method began to lose accuracy as the maximum slope of the wave became large, shown by slow decay of the modal coefficients. However, with 100 modes it was possible to calculate overhanging waves for values of $\rho_{2} / \rho_{1}$ greater than $0 \cdot 9$, but the accuracy was not good enough to follow such solutions to greater heights. The vortex-sheet method was much better for steep waves, and 65 intervals in $0<\alpha<\pi$ were sufficient to obtain results accurate to three significant digits for the highest wave calculated and demonstrate overturning for $\rho_{2} / \rho_{1}=0 \cdot 1$. The results described here were obtained by this method. Both methods were implemented on the Cray-1 at NCAR using 64 bit arithmetic.

From Holyer's results it is known that the phase speed is monotonic in the waveheight $a$, defined by

$$
\frac{1}{2}(y(0)-y(\pi))=a,
$$

until the point of overturning. However, since the behaviour of the phase speed with waveheight in the overturned region was unknown, and the possibility that it does not increase monotonically could not be ruled out, we provided the code with the 


$\begin{array}{ccc}\rho_{2} / \rho_{1} & a / \pi & a / \pi \\ & \text { (present results) } & \text { (from Holyer 1979 } \\ 0 \cdot 1 & 0 \cdot 220 \pm 0.003 & 0 \cdot 226 \pm 0 \cdot 006 \\ 0 \cdot 9 & 0 \cdot 348 \pm 0.003 & - \\ 1 \cdot 0 & 0 \cdot 353 \pm 0.003 & 0.350 \pm 0.006\end{array}$

TABr. 1. Wave heights at which surface first develops a vertical tangent
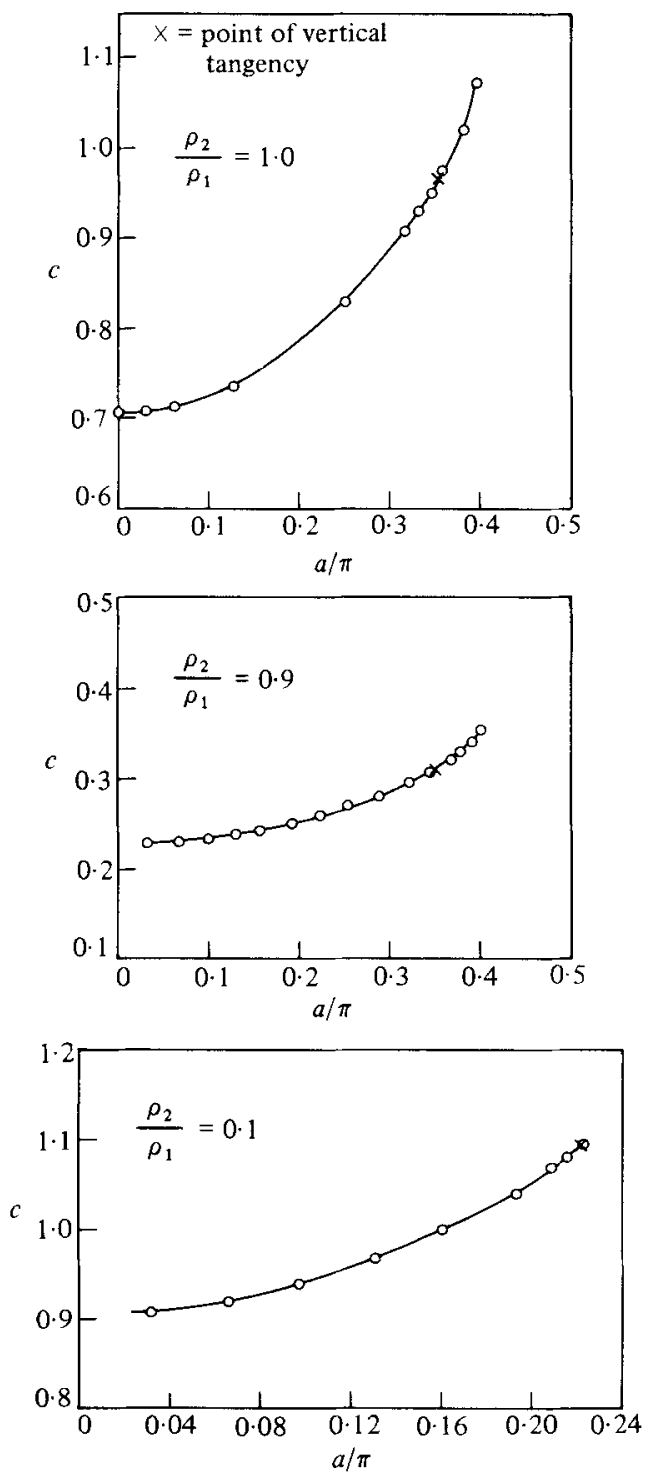

FIrure 1. Phase speed $c$ ss. wave steepness $a / \pi$ for $\rho_{2} / \rho_{1}=0 \cdot 1,0 \cdot 9,1 \cdot 0$. 


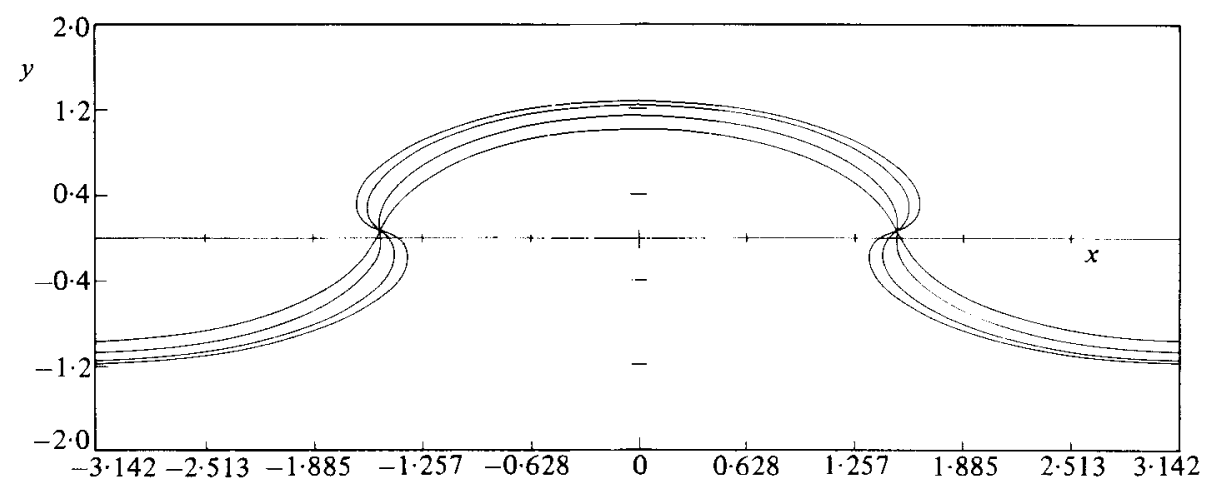

Figure 2. Profiles of steady interfacial waves for $\rho_{2} / \rho_{1}=0 \cdot 9$. The $x$-axis is the mean level.

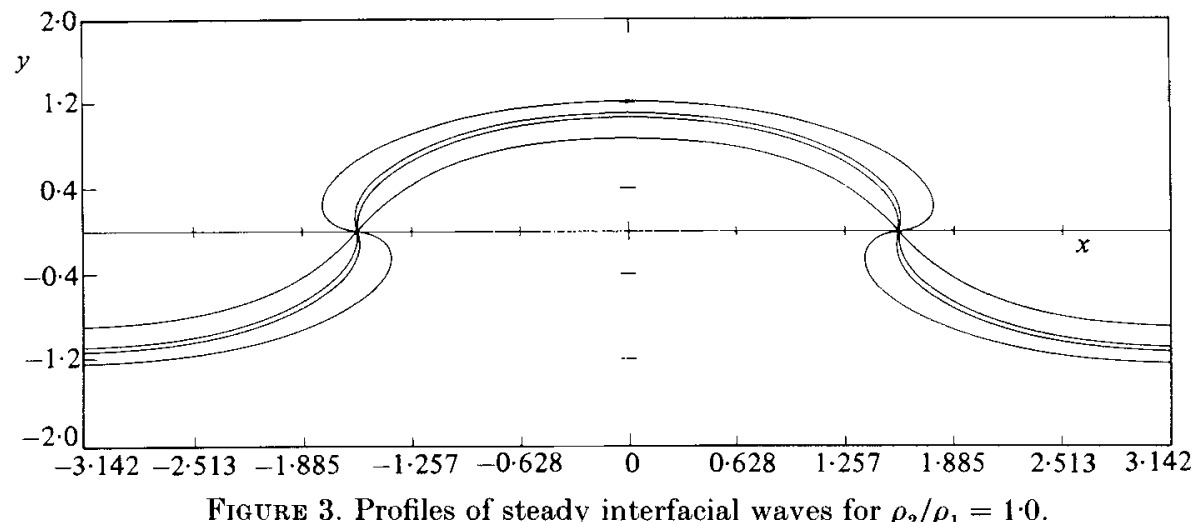

These waves are symmetrical about $y=0$.

option to compute the waveheight corresponding to a given phase speed. We show results for density ratios $\rho_{2} / \rho_{1}=0 \cdot 1,0 \cdot 9$, and $1 \cdot 0$. The last ratio corresponds to the Boussinesq limit, which is obtained formally by substituting $\left(\rho_{2}+\rho_{1}\right) y(x)$ for $\rho_{2} y(x)$ on the right-hand side of (2.2) and then putting $\rho_{2}=\rho_{1}$. This is equivalent to neglecting density differences except when multiplied by $g$.

The waveheights at which a vertical tangent first appears are given in table 1. For $\rho_{2} / \rho_{1}=0 \cdot 1$ and 1.0 they compare favourably with Holyer's estimates based on Padé approximants. The wave speed as a function of waveheight is plotted in figure 1 . It is seen that the waveheight continues to increase past the point of overturning. Thus the wave of greatest height does not correspond to the wave for which the profile first becomes vertical. As the phase speed was further increased the waveheight did begin to decrease. However, examination of the solutions corresponding to the decreasing wave height revealed the existence of high-frequency noise in the profile, a signal that the resolution used provided insufficient accuracy. As the calculations with 65 intervals require the inversion of a $194 \times 194$ matrix no effort was made to increase the resolution. Finally, in figures 2 and 3 we plot profiles of the vertical and overhanging waves for $\rho_{2} / \rho_{1}=0.9$ and 1.0 respectively. It was not possible to obtain this degree of overturning for the case $\rho_{2} / \rho_{1}=0.1$ as this value is close to the surface-wave limit and more points would be required in order to resolve the high curvatures corresponding to overhanging waves at this density ratio. 


\section{Conclusions}

In this paper we have provided numerical evidence for the existence of overhanging waves in which the $x$-velocity exceeds the phase speed of the wave in the overturned region. This evidence lends credibility to the conjecture of Saffman \& Yuen that such interfacial waves are most likely geometrically limited. That is, solutions continue to exist until the interface intersects itself. While we have been unable to compute this geometric limit, this work indicates that the criterion of vertical tangency does not correspond to a singular solution for interfacial waves. The stability of these steep waves to infinitesimal disturbances remains to be studied. It is interesting to note that the fluid particles on the interface between the points of vertical tangency in the overhang region are moving faster than the wave.

We thank Dr H. C. Yuen for the benefit of extensive discussions and valuable suggestions. This work was partially supported by the National Science Foundation (grant OCE-8100517). The Cray-1 at NCAR is supported by the National Science Foundation.

\section{REFERENCES}

Amick, C. J., Fraenkel, L. E. \& Toland, J. F. 1982 On the Stokes conjecture for the wave of extreme form. To appear.

Baker, G. R., Meiron, D. I. \& Orszag, S. A. 1982 Generalized vortex methods for free-surface flow problems. J. Fluid Mech. 123, 477-501.

Holyer, J. Y. 1979 Large amplitude progressive interfacial waves. J. Fluid Mech. 93, 433-448.

SaffMan, P. G. \& Yuen, H. C. 1982 Finite-amplitude interfacial waves in the presence of a current. J. Fluid Mech. 123, 459-476. 